\title{
Papuan Malay word stress reduces lexical alternatives
}

\author{
Constantijn Kaland ${ }^{1}$ and Vincent J. van Heuven ${ }^{2}$ \\ ${ }^{1}$ Institute of Linguistics, University of Cologne, Germany \\ ${ }^{2}$ University of Pannonia, Veszprém, Hungary \\ ckaland@uni-koeln.de, v.j.j.p.van.heuven@hum.leidenuniv.nl
}

\begin{abstract}
This study investigates the extent to which word stress facilitates word disambiguation in Papuan Malay. Although there is consistent acoustic support for word stress patterns in this language, the function of word stress in Indonesian languages, including Papuan Malay, has been disputed in several studies. Based on a word list of phonetically transcribed Papuan Malay words, an analysis of wordembeddings was carried out. The number of words that are embedded in other words was shown to explain the role of word stress in the word recognition processes crosslinguistically. The results of the lexical analysis indicate that Papuan Malay is somewhat similar to English, a language where word stress differences are mainly signalled by vowel quality and to a lesser extent by suprasegmental cues. The results are discussed within the context of cross-linguistic cues to word stress and shed a new light on the controversy concerning word stress in Indonesian languages.
\end{abstract}

Index Terms: word stress, word recognition, lexical analysis

\section{Introduction}

The literature has shown that word stress may facilitate word recognition. The extent to which word stress has this facilitation effect differs per language. For example, much depends on what acoustic cues are used to signal word stress and the extent to which these cues are suprasegmental (i.e. duration, intensity, spectral tilt) or segmental (i.e. vowel quality). It is furthermore known that not all languages of the world have stress. In stress-less languages, acoustic cues to word recognition are found at the segmental level and/or in lexically specified F0 patterns (tone languages). Of particular interest to this study is Papuan Malay, a language from the Trade Malay family [1], in which word stress has been claimed to be non-existent. The discussion in the literature relates to a larger one on the role of word stress in Indonesian languages. Although recent studies have found support for word stress in Papuan Malay, less is known its role in word recognition. The current study shows that this function of word stress is present in Papuan Malay.

\subsection{Word recognition and stress}

When perceiving speech, listeners face the task of matching the input signal with their mental lexicon. Models of speech perception are based on the idea that this matching process evolves by means of rejecting alternative words (e.g. [2], [3]). This means that multiple word candidates are simultaneously activated as soon as the speech signal cues them, i.e. upon perception of the first phonemes of a word. For example, the sequence [bæ] could activate candidate words such as band, bandit, baritone, backup etc., making the speech signal up to these two phonemes ambiguous. Thus, the listener's task is to select the best match between speech signal and a stored mental form of a word by means of disambiguation. This task is generally accomplished after all alternative candidates are rejected, which could be as early as after the first couple of phonemes or only after the entire word has been perceived. In the case of [bæ], which is a plausible onset of numerous English words, successful rejection of all alternatives is expected to occur after a larger portion of the word has been produced. Studies have shown that besides the phonemic information or contextual expectations, also prosody can facilitate word recognition processes [4]. In particular, it has been shown that word stress can be an effective cue to the rejection of alternative words during recognition.

Word stress is generally defined according to prominent suprasegmental features of one particular syllable in a word. For example, stressed syllables are generally longer, louder and higher pitched than unstressed syllables. Stressed syllables tend to follow (predictable) patterns in languages of the world and listeners generally rely on these patterns for word recognition. For example, it has been shown that Dutch listeners can successfully choose between orgel (penultimate stress; 'organ') and orkest (ultimate stress; 'orchestra') after hearing only the first syllable (e.g. [5]). In English, however, listeners had more difficulties selecting the correct word in a similar task [6]. These differences illustrate how word stress is perceived in these languages. The most reliable perceptual correlate of word stress in Dutch is duration (suprasegmental; e.g. [7]), and in English vowel quality (segmental; e.g. [8]). It has to be noted, though, that in the produced speech signal, either of these cues is present in either language. Thus, word recognition processes may differ substantially, even among closely related languages ([9],[10]).

\subsection{Lexical analyses}

Analyses on lexicon databases showed that typological differences in the recognition of stress patterns can be best explained by the number of embedded words in a language's lexicon ([10],[12],[13]). Polysyllabic words may have shorter words embedded in them, for example bee is embedded in the first syllable of beanie or belay. The mean number of embedded words per word differed considerably between English (0.49) and Spanish (2.32) when stress was not taken into account ([12],[13]), falsely suggesting that word recognition processes would face a larger challenge in the latter language. However, crucial for the embedding analysis is the difference between the total number of embeddings and the number of embeddings when stress is taken into account. Thus, when stress is taken into account, bee only counts as an embedding in beanie, where it corresponds to the stressed syllable, and not in belay where it corresponds to an unstressed syllable. Under the assumption that monosyllabic 
words are stressed, these counts revealed differences between English on the one hand and Dutch, German and Spanish on the other hand ([13], Table 1). That is, the relative decrease in mean embeddings per word due to the consideration of stress was the largest in Spanish and the smallest in English. Dutch and German occupied middle positions. For all of these languages the mean stress-matched embeddings was below the crucial limit of one per carrier word, showing that stress information successfully reduces the competition between carrier and embedding. This means that the disambiguation problem that listeners face, which strictly speaking only exists when there are embeddings (i.e. $\geq 1$ per carrier word), can be successfully minimized by suprasegmental stress cues ([9], [13]). For English, the statistics showed that even when stress information was ignored, the mean number of embeddings per carrier was below one. It was argued that the differences among the lexical statistics of each language could be explained by the type of stress cues listeners exploit in word recognition. In Spanish, listeners use mainly suprasegmental cues (see also [14]). In Dutch and German, both suprasegmental and segmental cues are used, whereas in English, segmental cues are most important [6].

Table 1. Mean number of embedded words per word when ignoring stress (left), when considering stress (mid), and the proportion of the latter (right) data

\begin{tabular}{lccc}
\multicolumn{4}{c}{ from [12] and [13]. } \\
\hline \hline & All emb. & Stress-matched & Proportion \\
\hline Dutch & 1.52 & 0.74 & 0.49 \\
English & 0.94 & 0.59 & 0.62 \\
German & 1.62 & 0.80 & 0.49 \\
Spanish & 2.32 & 0.73 & 0.31 \\
\hline
\end{tabular}

\subsection{Stress in languages of Indonesia}

Even if we leave tone languages aside, not all languages make use of stress distinctions. For example, some Indonesian languages have been reported to lack word stress. This was shown in a gating experiment in which listeners (Balinese, Sundanese and Javanese) needed to recognize phrase-final words based on fragments of increasing length (gates; [15]). The first gate corresponded to the first syllable of the word, the second gate corresponded to the first syllable and the part of the second syllable up to and including the vowel. The hypothesis that the first gate provided enough (suprasegmental) information to successfully recognize the word was rejected, indicating that the acoustic stress distinctions were not picked up by the Indonesian listeners. The same task was done by Dutch listeners and showed that they did make use of the stress cues in the first syllable. In another perception study, Indonesian listeners (regional language not reported) rated the position of the stressed syllable and the acceptability of Indonesian words with manipulated F0 patterns [16]. Although different words showed systematic differences in the location judgements, the acceptability ratings were similar for each stress position. This result lead to the conclusion that Indonesian word stress is not bound to a particular syllable in the word. It needs to be noted, however, that there is considerable variability among the languages of Indonesia [17]. This was shown in a perception experiment in which listeners of Toba Batak and Javanese rated the acceptability of different stress patterns [18]. Results indicated a clear preference for stress in penultimate position for Toba Batak listeners, and no preference for any of the positions for Javanese listeners.

Work on the Trade Malay languages, spoken in the Eastern part of Indonesia, reported contradicting findings on the production of word stress. For Ambonese, initial stressclaims [19] were rejected by an acoustic analysis [20]. Manado Malay was reported to have penultimate word stress, although no acoustic or perceptual verification for this claim has been carried out [21]. As for Papuan Malay, a perception study on phrase prominences and boundaries concluded that this language lacks word stress [22]. An acoustic analysis at the word level, however, showed that duration, spectral tilt and vowel reduction are the strongest correlates of word stress [23]. Furthermore, the only two mid-vowels in Papuan Malay $(/ \varepsilon /$ and $/ \mathrm{\rho} /)$ tend to reject the otherwise regular penultimate stress pattern consistently [24], indicating the importance of vowel quality in word stress placement. Preliminary perceptual evidence [25] suggested that listeners are particularly sensitive to the irregular (ultimate) stress pattern. It remains to be seen, however, to what extent word stress patterns facilitate word recognition in this language.

\subsection{Research aim}

To sum up, this aim of this study is to shed more light on the function of word stress patterns in Papuan Malay. Such an analysis could solve part of the controversy on the status of word stress in this language and in related languages. In particular, this paper addresses the contribution of word stress differences to the rejection of alternative words. To this end a lexical analysis of word embeddings was carried out, similar to [12] and [13]. As shown in the literature, such an analysis reveals the functional load of word stress in word recognition. The analysis is further described in the next section.

\section{Methodology}

To investigate the number of embedded words, a list of phonetically transcribed Papuan Malay words was used (Appendix A in [25]). The list consisted of a written lexeme, a phonetic transcription, a word class label and an English gloss. For the purpose of this study, only Papuan Malay roots were considered, i.e. excluding loanwords. Papuan Malay has a large number of loanwords and these were considered to be less representative for native word stress patterns, following previous studies (e.g. [23]). The phonetic transcriptions included diacritics and thus allow for a precise comparison between lexemes, representative of spoken language [25] Before obtaining the number of embedded words, duplicates (e.g. homonyms such as pasang for 'pair' or 'market') were removed from the word list such that only single instances of each word in the list were left. Given the paucity of foursyllable words in the list $(\mathrm{N}=3)$, they were excluded from the counting procedure.

Additional information per word was added to the list based on the phonetic transcriptions. That is, based on the syllable boundary indications in the phonetic transcriptions, the number of syllables was calculated per word. In addition, the stressed syllable was indicated as a number referring to the position of that syllable for each word, based on the stress marks in the phonetic transcriptions. The final word list used for analysis consisted of 1106 words of which 1062 polysyllabic words as potential candidates for carriers. Table 2 provides the word counts for the analysed word list. 
Table 2. Word list counts by number of syllables $(\sigma)$ and word stress.

\begin{tabular}{lccc}
\hline \hline Number of $\sigma$ & Penult stress & Ult stress & All stresses \\
\hline One & - & - & 44 \\
Two & 892 & 103 & 995 \\
Three & 63 & 4 & 67 \\
\hline Total & 955 & 107 & 1106 \\
\hline \hline
\end{tabular}

In [13], the mean embeddings were weighed by word frequency. For Papuan Malay no corpus data is available to provide word frequencies. In the current analysis, the embedding statistics are therefore unweighted (see section 4 for further discussion). The analysis carried out in this study is based on the word list described in Table 2. Although a fair number of words might not be frequently occurring in spontaneous speech, the word list still provides a subset that is representative for the language. That is, the words in the list were elicited in spontaneous conversations and formed the basis for phonological analyses in [25]. In addition, a possible confounding effect due to the lack of frequency data would affect the total number of embeddings and the subset of stressmatching embeddings in an equal way.

With the absence of frequency data, the current analysis established the number of polysyllabic words which contained one or more embeddings and counted the subset of these embeddings for which stress matched between carrier word and embedded word. Following previous lexical analysis studies, syllable boundaries were taken into account. For example, $k e$ 'to' would be counted as an embedding in kewa ('dance party'; penult stress), but not in kembang ('flower'; penult stress). As for stress-matching embeddings, $\mathrm{ka}$ 'or' would count in kali ('river'; penult stress) but not in muka ('front'; penult stress). The counts were carried out in Excel [27] using syllable-level string matching based on the phonetic transcriptions in the word list.

\section{Results}

Table 3. Word $(\omega)$ counts and embeddings for each $\omega$ length in syllables $(\sigma)$ in the Papuan Malay word list.

\begin{tabular}{lccc}
\hline \hline$\omega$-length $(\sigma)$ & $\omega$ with emb. & all emb. & stress-matched \\
\hline Two & 136 & 139 & 84 \\
Three & 23 & 31 & 18 \\
\hline All lengths & 159 & 170 & 102 \\
\hline \hline
\end{tabular}

As reported in Table 3, the list consisted of a total of 159 polysyllabic carrier words for which embedded words could be found (column " $\omega$ with emb."). The embedded words had a length of either one syllable (in carrier words of two or three syllables) or two syllables (in carrier word of three syllables). The total number of embedded words (column "all emb.") was overall slightly higher than the number of words with embeddings. This result indicated that a small number of carrier words had more than one embedding $(M=1.07)$. When counting only the embedded words that matched for stress with the carrier word (column "stress-matched"), numbers were slightly lower than the number of words with embeddings. The latter observation is an indication that when stress is taken into account, the mean number of embeddings per carrier word is below one $(M=0.64)$.
In addition, the location of the embedded word in the carrier word was counted, providing an insight into which syllables overlapped between the embedding and the carrier (see [12] for comparable tables with ratios). Table 4 and Table 5 report the locations for all embeddings and for stressmatched embeddings respectively. Both tables show that the decrease in embeddings due to the consideration of word stress was particularly large for disyllabic carrier words that had embeddings starting in the second syllable, and for trisyllabic carrier words that had embeddings starting in the first syllable. In the latter case, all embeddings $(\mathrm{N}=8)$ could be disambiguated on the basis of stress information.

Table 4. All embeddings: length and location of embedded word $(E)$ in carrier word $(C)$ for each carrier word length in syllables $(\sigma)$.

\begin{tabular}{ccccc}
\hline \hline \multicolumn{2}{c}{ Length $(\sigma)$} & \multicolumn{3}{c}{ Location of onset of E in C } \\
Carrier & Emb. & 1st $\sigma$ & 2nd $\sigma$ & 3rd $\sigma$ \\
\hline Two & 1 & 95 & 44 & - \\
\hline \multirow{2}{*}{ Three } & 1 & 8 & 8 & 3 \\
& 2 & 4 & 8 & 0 \\
\hline \hline
\end{tabular}

Table 5. Stress-matched embeddings: length and location of embedded word $(E)$ in carrier word $(C)$ for each carrier word length in syllables $(\sigma)$.

\begin{tabular}{ccccc}
\hline \hline \multicolumn{2}{c}{ Length $(\sigma)$} & \multicolumn{3}{c}{ Location of onset of E in C } \\
Carrier & Emb. & 1st $\sigma$ & 2nd $\sigma$ & 3rd $\sigma$ \\
\hline Two & 1 & 82 & 5 & - \\
\hline \multirow{2}{*}{ Three } & 1 & 0 & 8 & 0 \\
& 2 & 2 & 8 & 0 \\
\hline \hline
\end{tabular}

\section{Discussion}

The lexical analysis reported in this study shows that many word embeddings can be successfully eliminated during word recognition on the basis of mismatching stress patterns in Papuan Malay. The reduction is most clearly found for embeddings with an onset in an unstressed syllable of the carrier word (Table 4 and Table 5). This can be explained when considering that stress is highly regular in Papuan Malay (penultimate) and that most of the embeddings concern monosyllabic words. Although word frequency could not be taken into account in the current study, the mean number of embeddings per word (section 3) can now be tentatively compared with the data in Table 1. Papuan Malay, although with slightly higher mean values, appears to be most similar to English. For a more direct comparison between the Papuan Malay values and those in Table 1, the proportions of stressmatched embeddings can be computed by subtracting the mean value of stress-matched embeddings per carrier word from the mean value of all embeddings per carrier word. In this way, low proportions predict large facilitation, whereas high proportions predict small facilitation. These proportions thus give an insight into the relative magnitude of the facilitatory effect of stress on word recognition and abstracts over language specific numbers of embeddings (Table 1, right). The highest proportions are found for English (0.62) and Papuan Malay (0.60), followed by German and Dutch (each 0.49), whereas Spanish shows the lowest proportions of stress-matched embeddings (0.31).

The observations of this study lead to the conclusion that word stress in Papuan Malay has a potential function in word recognition in that it may aid the process of rejecting 
alternative word candidates. Given the similarity with the results of [12] and [13] for English, it needs to be further discussed to what extend the facilitatory effect of word stress in Papuan Malay can be found in suprasegmental or segmental cues. Although such a conclusion can be drawn more reliably from a perception experiment addressing each of these cues, the current results and the available literature allow for some speculation. It was found that vowel reduction in Papuan Malay is among the strongest acoustic correlates of word stress [23]. Perception experiments using manipulated stimuli showed that Papuan Malay listeners were mainly sensitive for the irregular penultimate stress pattern [25]. Crucially, in [25] the effect of vowel reduction on stress perception was not tested. It therefore remains to be seen to what extent vowel reduction can successfully distinguish stressed and unstressed syllables in perception. The current results suggest that this is a plausible option. That is, Papuan Malay patterns with English, a language in which suprasegmental cues to word stress are of secondary importance, which is reflected in the relatively small facilitative effect of stress information on alternative word candidate rejection. If suprasegmental stress information is indeed of smaller importance for word recognition compared to languages such Dutch, German or Spanish, a larger role could be reserved for vowel reduction in Papuan Malay.

Papuan Malay and English appear similar in both the magnitude of the stress facilitation (as shown by the proportions above) and their individual mean values of embeddings per carrier word (Table 1 and section 3 ). However, a crucial difference between Papuan Malay and English concerns the mean embeddings per carrier word when all embeddings are counted. This can be illustrated when recalling that one is the crucial limit for the (mean) number of embeddings per carrier word (Table 1, section 1.2). In English, the mean value is just below one (0.94), whereas in Papuan Malay this number is just above one (1.07). In English, therefore, disambiguation is less of a challenge for listeners to begin with. In Papuan Malay, however, there is more need to disambiguate than in English, predicting that the relative importance of suprasegmental cues is larger in the former language. It has to be noted that taking into frequency data could still change this number for Papuan Malay.

The total number of embeddings found in this study make up less than $15 \%$ of the word list. This means that for maximally twice that percentage (roughly all embeddings plus their carrier) there is a need for stress-based disambiguation. Thus, in Papuan Malay the segmental information is sufficient to recognize the majority $(>70 \%)$ of the words. The relative frequency of embeddings in the other languages discussed here is unavailable, although it is expected that prosodic cues are not as important as segmental cues for word recognition.

Concerning the controversy of word stress claims in Indonesian languages this study has provided new data in support of the stress claim for Papuan Malay. It remains to be seen how the relative importance of suprasegmental versus segmental cues affects perception, as discussed in the above. Nevertheless, given the acoustic support [23] and the potential disambiguation function shown in this study, it seems that word stress in Papuan Malay is at least as functional as in English, which is uncontroversially a stress-language. More word-recognition research on Papuan Malay is needed to confirm such a conclusion.

\section{Acknowledgements}

Research for this paper was funded by the German Research Foundation (DFG,) - Project-ID 281511265 - SFB 1252. The authors thank Anne Cutler and four anonymous reviewers for valuable comments.

\section{References}

[1] S. H. Paauw, The Malay contact varieties of eastern Indonesia: A typological comparison. 2009 .

[2] W. Marslen-Wilson and P. Warren, 'Levels of perceptual representation and process in lexical access: Words, phonemes, and features.', Psychological Review, vol. 101, no. 4, pp. 653$675,1994$.

[3] J. M. McQueen, A. Cutler, T. Briscoe, and D. Norris, 'Models of continuous speech recognition and the contents of the vocabulary', Language and Cognitive Processes, vol. 10, no. 34, pp. 309-331, 1995.

[4] A. Cutler, 'Lexical Stress', in The Handbook of Speech Perception, D. B. Pisoni and R. E. Remez, Eds. Oxford, UK: Blackwell Publishing Ltd, 2005, pp. 264-289.

[5] V. Van Heuven, 'Effects of stress and accent on the human recognition of word fragments in spoken context: Gating and shadowing', W.A. Ainsworth \& J.N. Holmes (eds.), Proceedings of the 7th FASE/Speech-88 Symposium, $811-818,1988$.

[6] N. Cooper, A. Cutler, and R. Wales, 'Constraints of Lexical Stress on Lexical Access in English: Evidence from Native and Non-native Listeners', Lang Speech, vol. 45, no. 3, pp. 207-228, 2002.

[7] A. M. C. Sluijter, V. J. van Heuven, and J. J. A. Pacilly, 'Spectral balance as a cue in the perception of linguistic stress', The Journal of the Acoustical Society of America, vol. 101, no. 1, pp. 503-513, 1997.

[8] B. D. Fear, A. Cutler, and S. Butterfield, 'The strong/weak syllable distinction in English', The Journal of the Acoustical Society of America, vol. 97, no. 3, pp. 1893-1904, Mar. 1995.

[9] A. Cutler, Native listening: language experience and the recognition of spoken words. Cambridge, UK: The MIT Press, 2012.

[10] A. Cutler, R. Wales, N. Cooper, and J. H. Janssen, "Dutch listeners' use of suprasegmental cues to English stress," in Proceedings of the 16th International Congress of the Phonetics Sciences, Saarbrücken, Germany, 2007, pp. 1913-1916.

[11] V. Van Heuven and P. J. Hagman, 'Lexical statistics and spoken word recognition in Dutch', in Linguistics in the Netherlands, P. Coopmans and A. Hulk, Eds. Dordrecht, The Netherlands: Foris, 1988, pp. 59-68.

[12] A. Cutler, D. Norris, and N. Sebastián-Gallés, 'Phonemic repertoire and similarity within the vocabulary', presented at the 8th International Conference on Spoken Language Processing (Interspeech 2004-ICSLP), 2004, pp. 65-68.

[13] A. Cutler and D. Pasveer, 'Explaining cross-linguistic differences in effects of lexical stress on spoken-word recognition', in Proceedings of Speech Prosody 2006, Dresden, Germany, 2006, p. paper 250.

[14] S. Peperkamp, I. Vendelin, and E. Dupoux, 'Perception of predictable stress: A cross-linguistic investigation', Journal of Phonetics, vol. 38, no. 3, pp. 422-430, 2010.

[15] E. Van Zanten and V. Van Heuven, 'Word stress in Indonesian; Its communicative relevance', Bijdragen tot de Taal-, Land- en Volkenkunde / Journal of the Humanities and Social Sciences of Southeast Asia and Oceania, vol. 154, 1998.

[16] E. Van Zanten and V. J. Van Heuven, 'Word stress in Indonesian: Fixed or free?', NUSA Linguistic Studies of Indonesian and other Languages in Indonesia, vol. 53, pp. 1-20, 2004.

[17] N. P. Himmelmann and D. Kaufman, 'Prosodic systems: Austronesia', in The Oxford Handbook of Language Prosody, vol. IV Prosodic systems - areal groupings, C. Gussenhoven and A. Chen, Eds. in press. 
[18] R. W. N. Goedemans and E. van Zanten, 'Stress and accent in Indonesian', in Prosody in Indonesian Languages, vol. 9, van Heuven, Vincent J. and van Zanten, E, Eds. 2007, pp. 35-62.

[19] D. Van Minde, Malayu Ambong: Phonology, Morphology, Syntax. Research School CNWS, 1997.

[20] R. Maskikit-Essed and C. Gussenhoven, 'No stress, no pitch accent, no prosodic focus: the case of Ambonese Malay', Phonology, vol. 33, no. 2, pp. 353-389, 2016.

[21] R. B. Stoel, 'The intonation of Manado Malay', in Prosody in Indonesian Languages, vol. 9, van Heuven, Vincent J. and van Zanten, E, Eds. 2007, pp. 117-150.

[22] S. Riesberg, J. Kalbertodt, S. Baumann, and N. P. Himmelmann, 'On The Perception Of Prosodic Prominences And Boundaries In Papuan Malay', in Perspectives on information structure in Austronesian languages, S. Riesberg, A. Shiohara, and A. Utsumi, Eds. Berlin, Germany: Language Science Press, 2018, pp. 389-414

[23] C. C. L. Kaland, 'Acoustic correlates of word stress in Papuan Malay', Journal of Phonetics, vol. 74, pp. 55-74, 2019.

[24] C.C.L. Kaland, N. P. Himmelmann, and A. Kluge, 'Stress predictors in a Papuan Malay random forest', in Proceedings of the 19th International Congress of Phonetic Sciences, Melbourne, Australia, 2019, pp. 2871-2875.

[25] C. C. L. Kaland, "Offline and online processing of acoustic cues to word stress in Papuan Malay," The Journal of the Acoustical Society of America, vol. 147, no. 2, pp. 731-747, Feb. 2020, doi: $10.1121 / 10.0000578$.

[26] A. Kluge, A grammar of Papuan Malay. Berlin, Germany: Language Science Press, 2017.

[27] Microsoft Corporation. (2016). Microsoft Office Professional Excel (Version 16.0.4266.1001). 